\title{
Aberrant Differentiation of Human Pluripotent Stem Cell-Derived Kidney Precursor Cells inside Mouse Vascularized Bioreactors
}

\author{
Parisa Ranjzad ${ }^{a} \quad$ Jessica Jinks ${ }^{a} \quad$ Amir P. Salahia loannis Bantounas ${ }^{a}$ \\ Brian Derby ${ }^{b}$ Susan J. Kimber ${ }^{a}$ Adrian S. Woolfa, c Jason K.F. Wong ${ }^{\text {a, }}$ \\ ${ }^{a}$ Division of Cell Matrix Biology and Regenerative Medicine, School of Biological Sciences, Faculty of Biology \\ Medicine and Health, University of Manchester, Manchester, UK; ${ }^{b}$ Department of Materials, School of Natural

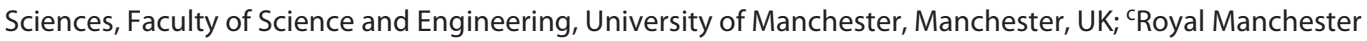 \\ Children's Hospital, Manchester University NHS Foundation Trust, Manchester Academic Health Science Centre, \\ Manchester, UK; dDepartment of Burns and Plastic Surgery, Manchester University NHS Foundation Trust, \\ Manchester Academic Health Science Centre, Wythenshawe Hospital, Manchester, UK
}

\section{Keywords}

Artery - Dysplasia · Flow-through · Glomerulus · Muscle · Tubule $\cdot$ Vein $\cdot$ Vessel

\begin{abstract}
Background: Numerous studies have documented the in vitro differentiation of human pluripotent stem cells (hPSCs) into kidney cells. Fewer studies have followed the fates of such kidney precursor cells (KPCs) inside animals, a more lifelike setting. Here, we tested the hypothesis that implanting hPSC-derived KPCs into an in vivo milieu surgically engineered to be highly vascular would enhance their maturation into kidney tissues. Methods: 3D printed chambers containing KPCs were implanted into the thighs of adult immunodeficient mice. In some chambers, an arterial and venous flowthrough (AVFT) was surgically fashioned. After 3 weeks and 3 months, implants were studied by histology, using qualitative and quantitative methods. Results: After 3 weeks, chambers containing AVFTs were richer in small vessels than contralateral chambers without AVFTs. Glomeruli with capillary loops and diverse types of tubules were detected in all chambers. At 3 months, chambers contained only rudimentary tubules
\end{abstract}

and glomeruli that appeared avascular. In chambers with AVFTs, prominent areas of muscle-like cells were also detected near tubules and the abnormal tissues immunostained for transforming growth factor $\beta 1$. These features have similarities to renal dysplasia, a typical histological signature of human congenital kidney malformations. Conclusions: This study urges a note of caution regarding the in vivo fates of hPSC-derived kidney precursors, with pathological differentiation appearing to follow a period of increased vascularity.

(c) 2020 The Author(s)
Published by S. Karger AG, Basel

\section{Introduction}

The potential to generate kidney tissues from human pluripotent stem cells (hPSCs) promises to help understand the biology of healthy and diseased organs and may eventually lead to regenerative medicine therapies [1-4]. It is now well established that PSC-derived tissues can be maintained for several weeks in 3D cultures as "organoids" that contain nephron components, including immature glomeruli and diverse kidney tubules [5-10]. Far fewer studies have followed the fates of hPSC-derived kidney precursor cells

$\begin{array}{ll}\text { karger@karger.com } & \begin{array}{l}\text { (c) 2020 The Author(s) } \\ \text { Published by S. Karger AG, Basel }\end{array} \\ \text { www.karger.com/nef } & \begin{array}{l}\text { This article is licensed under the Creative Commons Attribution 4.0 } \\ \text { International License (CC BY) (http://www.karger.com/Services/ }\end{array} \\ \text { OpenAccessLicense). Usage, derivative works and distribution are } \\ \text { permitted provided that proper credit is given to the author and the } \\ \text { original publisher. }\end{array}$

Adrian S. Woolf

School of Biological Sciences, University of Manchester

Room D2515, Michael Smith Building Oxford Road

Manchester M13 9PT (UK)

adrian.woolf@manchester.ac.uk 
(KPCs) inside animals, a more life-like setting, where longer follow-up may be feasible compared with in vitro experiments. As examples, it was reported that more mature glomerular differentiation, including formation of glomerular capillary loops, can be achieved by implanting hPSCderived kidney tissues either subcutaneously $[8,11]$ or under the kidney capsule $[12,13]$ in immunocompromised mice. Unfortunately, there are drawbacks $[3,14]$ to current in vitro and in vivo technologies, including the limited size and vascularity attained by PSC-derived kidney tissues.

Using tissue engineering, so-called "vascularized bioreactors" can be created by surgically fashioning either an arteriovenous loop, in which an artery is directly connected with a vein, or an arterial and venous flow-through (AVFT) [15-18]. In the latter technique, a space is cleared between an artery and its adjacent vein, and these 2 vessels are laid inside a chamber, perforated to allow their ingress and egress. When chambers containing AVFTs were filled with Matrigel ${ }^{\mathrm{TM}}$, an extracellular matrix gel, they were reported to become populated by adipose tissue rich in blood vessels [18]. Furthermore, immature nonrenal cells, such as fetal hepatocytes $[15,16]$, have been reported to differentiate in a realistic manner when placed inside such vascularized bioreactors in vivo.

Here, we designed and undertook experiments to answer 2 questions. First, could an AVFT be fashioned in the thigh of an immunocompromised mouse and could it be maintained in this location inside a perforated 3D chamber? We show that this technical approach is feasible and that, assessed at 3 weeks, it results in a highly vascular environment. Second, would this scenario enhance the growth and differentiation of hPSC-derived KPCs placed inside such a chamber containing an AVFT? We found that implanted cells had formed glomeruli and tubules when assessed at 3 weeks but, unexpectedly, they subsequently came to resemble malformed kidney tissues when assessed at 3 months.

Fig. 1. Implantation of $3 \mathrm{D}$ printed chambers without human KPCs. a In each mouse, an AVFT (red, artery; blue, vein) was fashioned in 1 thigh, while the other thigh served as a control with no AVFT. b An AVFT being fashioned. c The 2 halves of the chamber in situ before being closed; note the lower half contains the AVFT and the central part of each half chamber contains a half pillar (small circle) that will form a single pillar when the chamber is closed. d-i Histology from chambers harvested after 3 weeks. d Cross section of a chamber with an AVFT stained with hematoxylin and eosin. Note that a indicates artery; $v$, vein; and $p$, the central pillar. Asterisks indicate sections of the wall (white) of the chamber; note the wall appears discontinuous because of the perforations. e Cross section of a chamber that did not receive an

\section{Results}

\section{Establishing AVFTs in Thighs of Immunodeficient}

Mice

In the first set of experiments, 3D printed perforated plastic chambers filled with Matrigel ${ }^{\mathrm{TM}}$, but not containing human cells, were surgically placed into the thighs of 6 adult female severe combined immunodeficiency beige (SCID/beige) mice, as detailed in the Materials and Methods. In 1 thigh of each mouse, an AVFT was fashioned to run through the gel filled chamber, and a gel filled chamber that lacked an AVFT was implanted into the opposite thigh of each mouse (Fig. 1a-c). Harvested after 3 weeks, the chambers were recovered from all 12 thighs (Fig. 1d, e). Histology revealed patent arteries and veins inside all 6 chambers with AVFTs, and the linings of these arteries (Fig. 1f) and veins (Fig. 1g) immunostained for the endothelial marker cluster of differentiation 31 (CD31), also known as platelet endothelial cell adhesion molecule. Small-calibre vessels expressing CD31 were detected inside all chambers, in both those with (Fig. 1h) and without (Fig. 1i) AVFTs, but they were not quantified. Some of these small-calibre vessels were detected close to arteries and veins (Fig. 1f, g). As expected, no kidney-like tissues, such as glomeruli or tubule-like structures, were detected inside any of the chambers.

\section{Generating Human KPCs in Culture for Implantation into Mice}

Having established the feasibility of fashioning AVFTs in SCID/beige mice, this technique was modified to study the effects on human hPSC-derived KPCs. In these mice, the SCID mutation causes immunodeficiency affecting B and $\mathrm{T}$ lymphocytes, while the beige mutation results in defective natural killer cells. Our previous studies of hPSC-derived KPCs implanted subcutaneously in the backs of similar mice $[8,11]$ found no rejection of the hu-

AVFT stained with hematoxylin and eosin. Asterisks indicate the wall of the chamber. $\mathbf{f}$ High-power view of artery in chamber with an AVFT immunostained with CD31 antibody (brown colour) and counterstained with hematoxylin. $\mathbf{g}$ High-power view of vein in chamber with an AVFT immunostained with CD31 antibody (brown colour) and counterstained with hematoxylin. h Highpower of small CD31 immunostained (brown) vessels in a chamber containing an AVFT. i High power of small CD31 immunostained (brown) vessels in a chamber that did not receive an AVFT. Bars in histology sections are $500 \mu \mathrm{m}$ in $\mathbf{d}$, e; $100 \mu \mathrm{m}$ in $\mathbf{f}, \mathbf{g}$; and $200 \mu \mathrm{m}$ in $\mathbf{h}$, i. KPC, kidney precursor cells; AVFT, arterial and venous flow-through.

(For figure see next page.) 


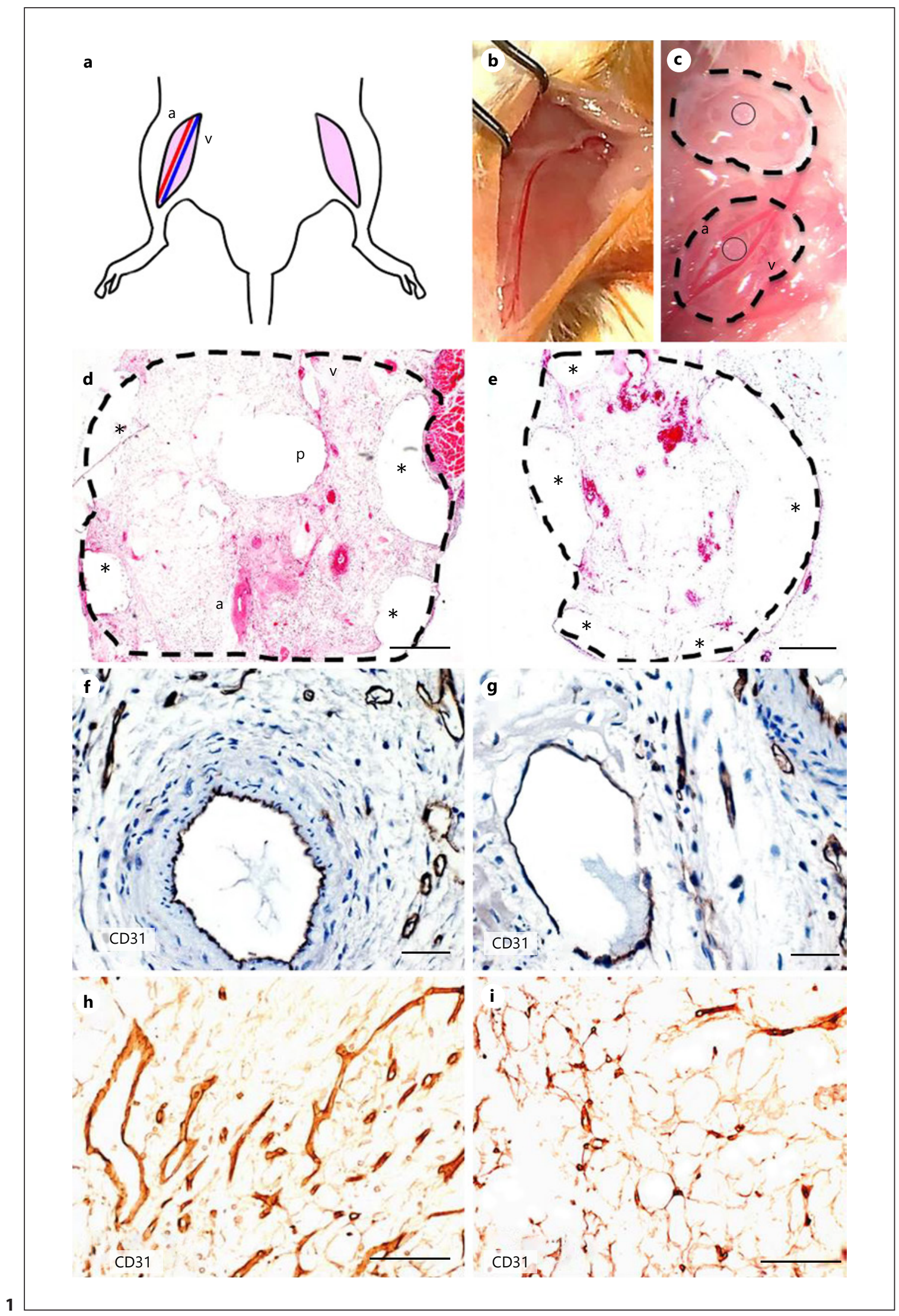



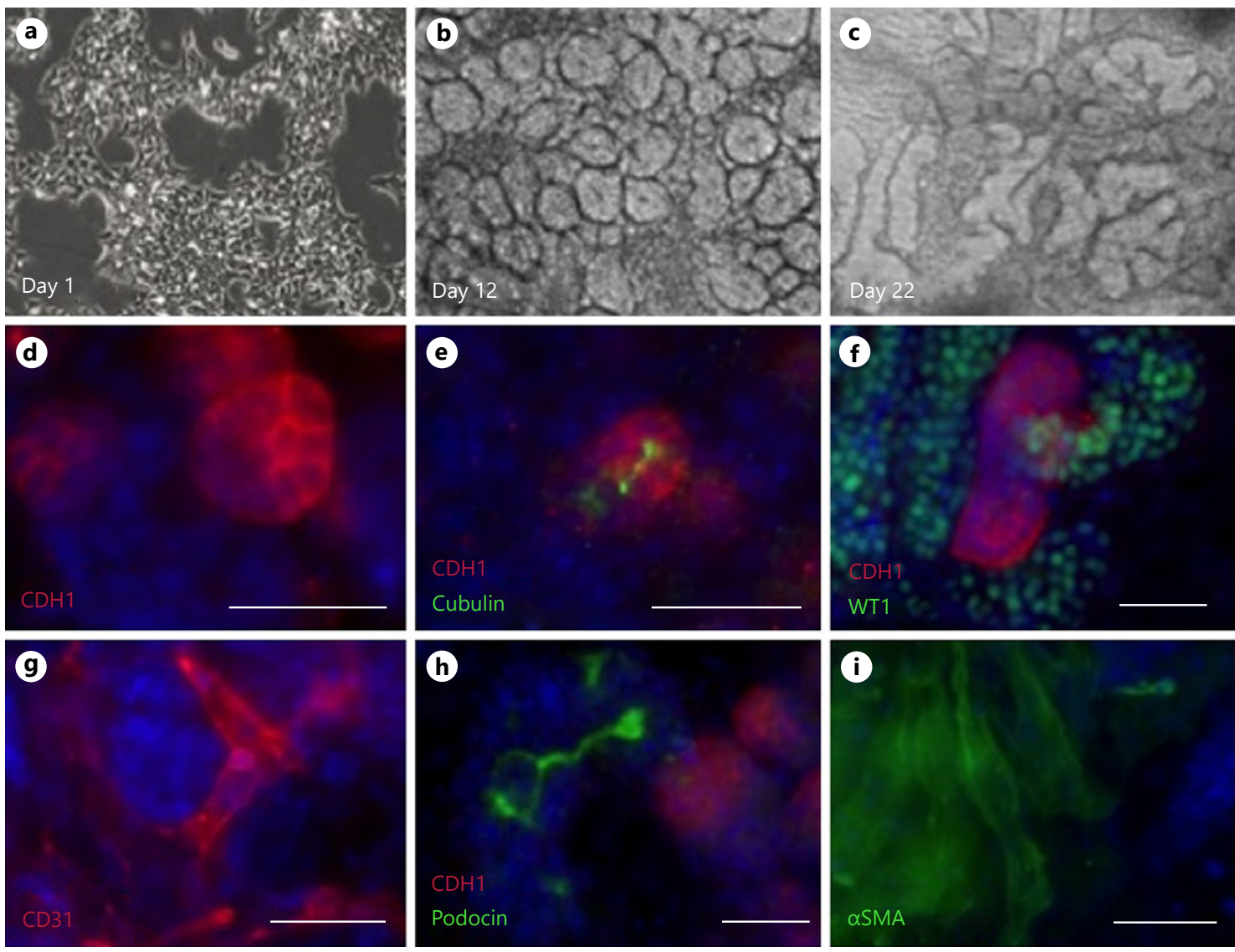

Fig. 2. In vitro differentiation of hPSCs to KPCs. a-c Phase contrast views of cultures at 1,12 , and 22 days of the kidney differentiation protocol. Note that the lawns of cells (day 1) acquired increasing complex morphology at 12 days (at which point, some of these KPC cultures were dissociated and implanted) and 22 days. d-i Immunocytochemistry at 32 days after start of in vitro differentiation with DAPI (blue) nuclear counterstain; all bars are 500 um d A CDH1 (red) immunostained tubule-like structure. e A tubule-like structure double immunostained for CDH1 (red) and cubilin (green). f A tubule-like structure immunostained for
CDH1 (red) surrounded by condensations of cells positive for WT1 (green). g Rudimentary capillary-like structures immunostained with anti-human CD31 (red). $\mathbf{h}$ This field shows a tubulelike structure immunostained for CDH1 (red) near a collection of cells that immunostained for podocin (green); note that the latter did not have an overt glomerular-like structure. i Zones of cells immunostained for a-SMA (green). hPSCs, human pluripotent stem cells; KPC, kidney precursor cells; CDH1, cadherin 1; WT1, Wilms tumour 1; $\alpha$-SMA, $\alpha$-smooth muscle actin.

Medium was used instead of the now discontinued STEMdiff ${ }^{\mathrm{TM}}$ APELTM Medium. Similar to our published observations using the former media [8], cultures at day 12 of the current protocol contained aggregates of cells (Fig. 2b), resembling cell clusters that we reported to express markers of nephron, stromal and ureteric bud lineages [8]. At this point, some cultures were enzymatically dissociated into single cells, and a pellet of $2 \times 10^{6}$ of these KPCs was embedded in Matrigel ${ }^{\mathrm{TM}}$ inside each chamber to be implanted into SCID/beige mice. Other parallel cultures were maintained in 2D for another 3 weeks. As expected [8], they began to form tubule-like structures (Fig. 2c). Cultures were examined by immunocytochem- 
Table 1. Antibodies used in this study

\begin{tabular}{|c|c|c|c|}
\hline Antibody & Host & $\begin{array}{l}\text { Commercial source and catalogue } \\
\text { number }\end{array}$ & $\begin{array}{l}\text { Application } \\
\text { and dilution }\end{array}$ \\
\hline a-SMA (reacts with mouse and human tissue) & Mouse & Sigma A2547 & $\begin{array}{l}\text { IHC } 1: 16,000 \\
\text { ICC } 1: 16,000\end{array}$ \\
\hline CD31 (reacts with human but not mouse tissue) & Mouse & Cell Signalling 3528 & $\begin{array}{l}\text { IHC } 1: 100 \\
\text { ICC } 1: 100\end{array}$ \\
\hline CDH1 (reacts with mouse and human tissue) & Mouse & Abcam 76055 & $\begin{array}{l}\text { IHC } 1: 5,000 \\
\text { ICC } 1: 400\end{array}$ \\
\hline Cubilin (reacts with human tissue) & Goat & Santa Cruz Biotechnology sc-20607 & $\begin{array}{l}\text { IHC } 1: 100 \\
\text { ICC } 1: 200\end{array}$ \\
\hline Synaptopodin (reacts with mouse and human tissue) & Mouse & Santa Cruz Biotechnology sc-515842 & IHC 1:200 \\
\hline TGF- $\beta 1$ (reacts with mouse and human tissue) & Mouse & Santa Cruz Biotechnology sc-130348 & IHC 1:20 \\
\hline UMOD (reacts with mouse and human tissue) & Rabbit & Santa Cruz Biotechnology sc-20631 & IHC $1: 100$ \\
\hline WT1 (reacts with mouse and human tissue) & Rabbit & Calbiochem CA1026 & IHC $1: 1,000$ \\
\hline WT1 (reacts with mouse and human tissue) & Rabbit & Santa Cruz Biotechnology sc-192 & ICC $1: 100$ \\
\hline
\end{tabular}

$\alpha$-SMA, $\alpha$-smooth muscle actin; CDH1, cadherin 1 ; HNF1B, hepatocyte nuclear factor $1 \mathrm{~B}$; TGF- $\beta 1$, transforming growth factor- $\beta 1$; UMOD, uromodulin; WT1, Wilms tumour 1; ICC, immunocytochemistry; IHC, immunohistochemistry.

istry at 32 days after the start of the differentiation protocol at the limit of the culture period. Subsets of tubules were positive for cadherin 1 (CDH1) (Fig. 2d), also known as E-cadherin, a collecting duct and distal tubule marker, or for cubilin (Fig. 2e), a proximal tubule marker. Subsets of nearby cells were reactive with antibodies to the nephron lineage protein Wilms tumour 1 (WT1) (Fig. 2f); CD31 (Fig. 2g), using an antibody that detects human protein (Table 1); podocin, a podocyte protein (Fig. 2h); or $\alpha$-smooth muscle actin ( $\alpha$-SMA) (Fig. 2i). Neither the WT1- nor the podocin-expressing cells, however, formed morphologically recognizable glomeruli in this culture setting, a similar result to our previous experiments with this hPSC line [8].

\section{Human KPC Implants Harvested 3 Weeks after Surgery}

All KPC implant sites (i.e., in the 12 thighs of the 6 mice) harvested 3 weeks after surgery contained cham- bers (Fig. 3a, b), and a patent artery and vein were detected by histology inside each chamber in which an AVFT had been fashioned (Fig. 3a). Small-calibre CD31 expressing vessels were detected inside all chambers, and they appeared more prominent in chambers with AVFTs (low power views, Fig. 3a, b, and high power view, Fig. 3c, d). Quantification of vessels, expressed as the percentages of cross-sectional areas occupied by CD31-positive structures, was undertaken. For each chamber, the quantified area comprised that enclosed by the chamber wall plus areas within the perforations of each chamber; tissues external to the chamber wall were excluded from analysis. For each chamber, a section from every 15 th serial slide was quantified in this manner, and all areas were summated to generate 1 consolidated result per chamber. Typically, using this method, around $35 \mathrm{~mm}^{2}$ was assessed per chamber, a summation of around 10 individual sections. Vessel density was statistically significantly $(p=0.031)$ higher in the chambers with AVFTs than in 


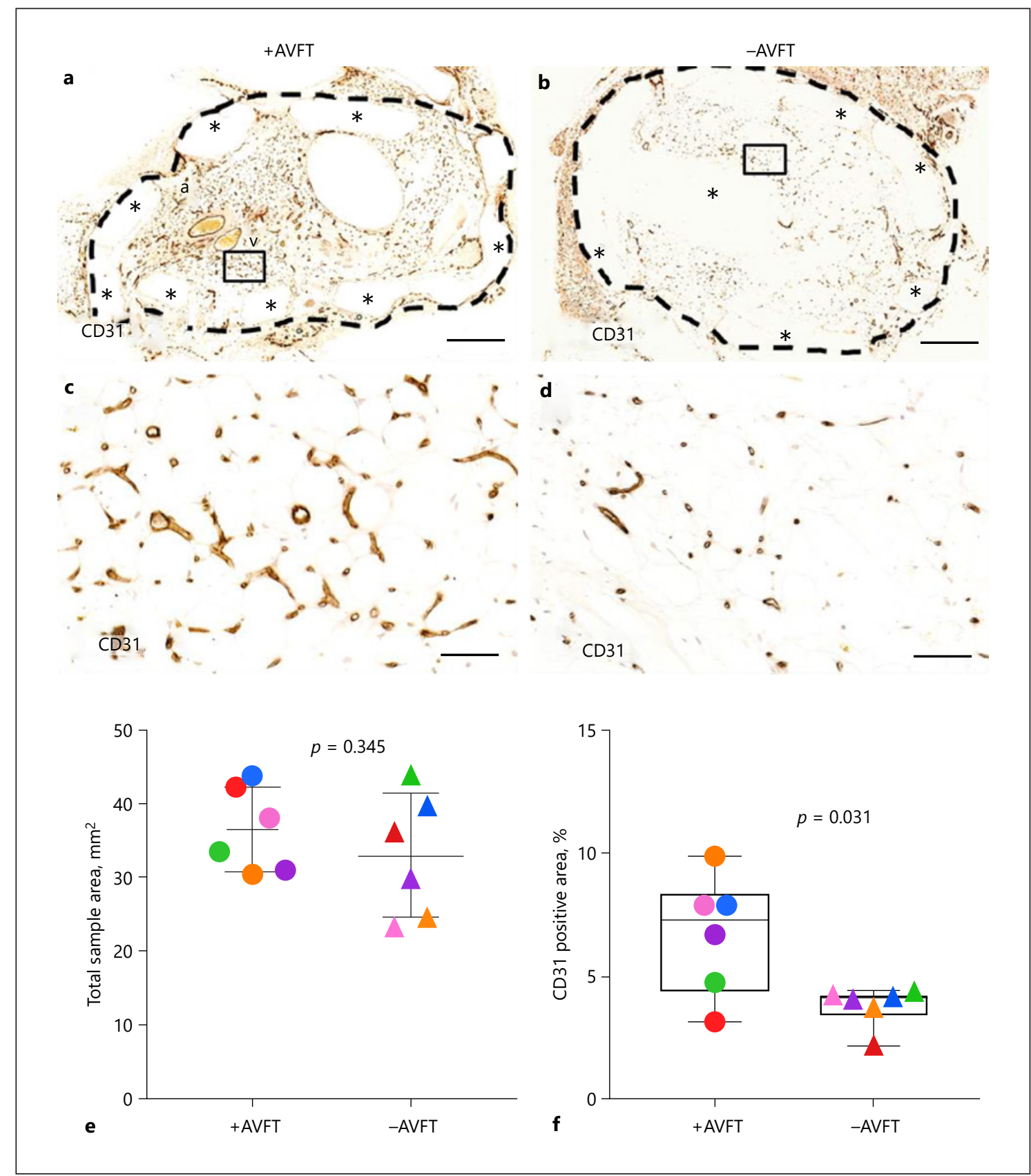

Fig. 3. Identification and quantification of vessels in chambers implanted with human KPCs and harvested after 3 weeks. a-d Histology sections immunostained for CD31 (brown) of chambers harvested 3 weeks after surgery. Bars are $500 \mu \mathrm{m}$ in (a, b) and $50 \mu \mathrm{m}$ in $(\mathbf{c}, \mathbf{d})$. a Low power of cross section of chamber with an AVFT. The dotted line is drawn around the outside of the chamber and the asterisks indicate chamber walls. $\mathrm{v}$ and $\mathrm{a}$ indicate a vein and an artery, respectively. Note that the brown CD31 signals are discerned. b Low power of cross section of chamber that lacks an AVFT. Note the lack of an artery and a vein and the fact that CD31 signals are hardly discerned at this low power. The dotted line is drawn around the outside of the chamber and the asterisks indicate walls and the central pillar of the chamber. c High power of dense small vessels from chamber with an AVFT. d High power of apparently more sparse small vessels in chamber lacking an AVFT. e Similar total areas used for CD31 quantification in chambers with AVFTs (left column) and those lacking AVFTs (right column); note that each mouse has a designated colour with the AVFT chamber shown as a circle and the opposite non-AVFT chamber shown as a triangle. $\mathbf{f}$ A significantly greater proportion of the chamber area was occupied by vessels, as assessed by CD31 immunostaining, in chambers with AVFTs compared with chambers lacking AVFTs. Of note, we did not observe direct continuity between the AVFT vessels and the small CD31 immunostaining vessels around them. KPC, kidney precursor cells; AVFT, arterial and venous flow-through. 
chambers without AVFTs (Fig. 3e, f). The total areas assessed for these analyses were similar $(p=0.345)$ for chambers with or without AVFTs. Of note, we did not observe direct continuity between the AVFT vessels and the small CD31 immunostaining vessels around them.
As visualized after hematoxylin and eosin staining, kidney-like tissues were apparent in all 12 chambers (Fig. 4a, b); no such tissues were detected outside the chamber walls. On closer inspection, these kidney-like tissues contained tubule-like and glomerular-like struc-

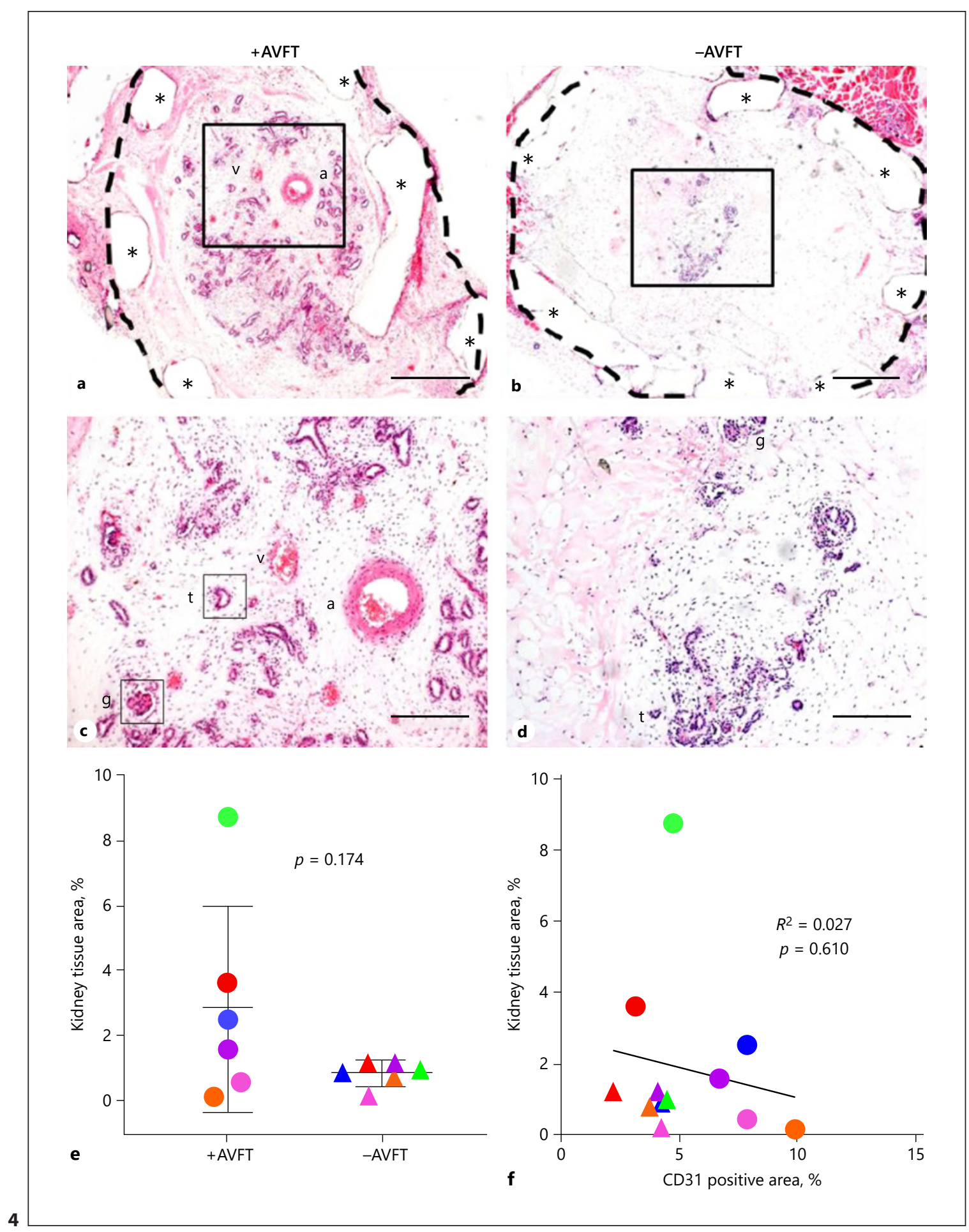

(For legend see next page.) 
tures (Figs. 4c, d, 5). Next, we measured the amount of "kidney tissue" in each chamber, as assessed by the summated areas occupied by tubules and glomeruli factored for the total areas assessed. There was no significant ( $p=$ 0.174) difference between inside the chambers with AVFTs compared with chambers without AVFTs (Fig. 4e). Considering all 12 chambers, there was no statistically significant $(p=0.610)$ correlation between the amount of CD31-positive vessels and the amount of kidney tissues (Fig. 4f).

Further inspection and immunohistochemical analyses revealed similar types of renal structures in chambers with or without AVFTs. These included glomeruli with red blood cells in their tufts (Fig. 5a). Cells in the Bowman capsule and peripheral glomerular tuft cells were of human origin as assessed by immunostaining for human mitochondrial protein (HuMt) (Fig. 5b and its inset). CD31-positive cells were detected inside glomerular tufts (Fig. 5c). Subsets of glomerular cells immunostained for the podocyte markers WT1 (Fig. 5d), synaptopodin (Fig. 5e), and podocin (Fig. 5f). Also detected were tubules (Fig. $5 \mathrm{~g}$ ) immunostaining for HuMt (Fig. 5h); as we had noted in subcutaneous implant experiments [8], tu-

Fig. 4. Identification and quantification of kidney-like tissues within chambers implanted with human KPCs and harvested after 3 weeks. a-d Histology sections stained with hematoxylin and eosin of chambers harvested 3 weeks after surgery. Bars are $500 \mu \mathrm{m}$ in $(\mathbf{a}, \mathbf{b})$ and $200 \mu \mathrm{m}$ in $(\mathbf{c}, \mathbf{d})$. a Low power of cross section of chamber with an AVFT. The dotted line is drawn around the outside of the chamber and the asterisks indicate walls of the chamber. a and $\mathrm{v}$ indicate an artery and a vein, respectively. The kidney-like structures appear blue and an enlargement of the boxed area is depicted in c, where tubule-like (t) and glomerular-like (g) structures separated by loose stromal-like tissues are noted. $\mathbf{b}$, $\mathbf{d}$ respectively show an overview and enlarged section of a chamber lacking an AVFT. Note that this chamber also contains tubules and glomer- bular immunostaining was more intense than in other cells such as presumed podocytes, most likely because tubules are enriched for mitochondria. Subsets of tubules immunostained for cubilin (Fig. 5i); uromodulin (Fig. 5j), a marker of the thick ascending limb of the loop of Henle; or CDH1, a marker of distal tubules and collecting ducts (Fig. 5k). No signals were detected upon omitting the primary antibodies (Fig. 5l). The CD31 antibody used in the above experiments reacts with both mouse and human protein, so we also probed sections with a CD31 antibody (see Table 1 for details) that is reactive with human but not mouse protein. From these experiments, we concluded that CD31 expressing vessels in glomerular tufts within implant chambers were likely to be of mouse origin (online suppl. Fig. 1; for all online suppl. material, see www.karger.com/doi/10.1159/000509425).

\section{Human KPC Implants Harvested at 3 Months after Surgery}

The other 6 mice were followed for 3 months after implantation of the same batches of human KPCs. All 12 chambers were recovered and analysed by histology (Fig. 6a-f), and all 6 chambers in which an AVFT had

uli. e Quantification of "kidney tissues" as assessed by the areas occupied by both tubules and glomeruli but excluding intervening stroma. The graph shows a non-significant tendency for more "kidney tissues" in chambers with AVFTs (left column) compared with those without AVFTs (right column). f Considering all 12 chambers with KPC implants harvested at 3 weeks, there was no significant correlation between percentage areas occupied by "kidney tissues" and CD31 structures. In the 2 graphs, note that each mouse has a designated colour with the AVFT chamber shown as a circle and the opposite non-AVFT chamber shown as a triangle. KPC, kidney precursor cells; AVFT, arterial and venous flowthrough.

prominent in presumed podocytes. e Synaptopodin immunostaining (brown). f Podocin immunostaining, with a linear pattern at the base of presumed podocytes; with this antibody, there is some background staining in surrounding tissues. g Tubule-like structures. $\mathbf{h}$ HuMt immunostaining (brown) in cytoplasm of tubules. i A subset of tubules (red arrows) react (brown) with the cubilin antibody. $\mathbf{j}$ Rare tubules (red arrows) react (brown) with the UMOD antibody. k Tubules (red arrows) reacting (brown) with the CDH1 antibody. I No brown colour when the primary antibody is omitted. KPC, kidney precursor cells; HuMt, human mitochondrial protein; WT1, Wilms tumour 1; UMOD, uromodulin; $\mathrm{CDH} 1$, cadherin 1.

(For figure see next page.) 
been fashioned contained a large artery and vein. The histological appearances of all 6 chambers that contained AVFTs (Fig. 6a, c, e) were similar but were strikingly different when compared with chambers lacking AVFTs (Fig. 6b, d, f). As visualized after hematoxylin and eosin staining, chambers with AVFTs were dominated by areas of elongated cells, which appeared muscle-like, and there were also primitive appearing tubules and avascular glomeruli. Chambers lacking AVFTs also contained primitive tubules, avascular glomeruli, and stromal-like cells but were not dominated by the muscle-like cells. As noted below, the muscle-like areas were of human origin,

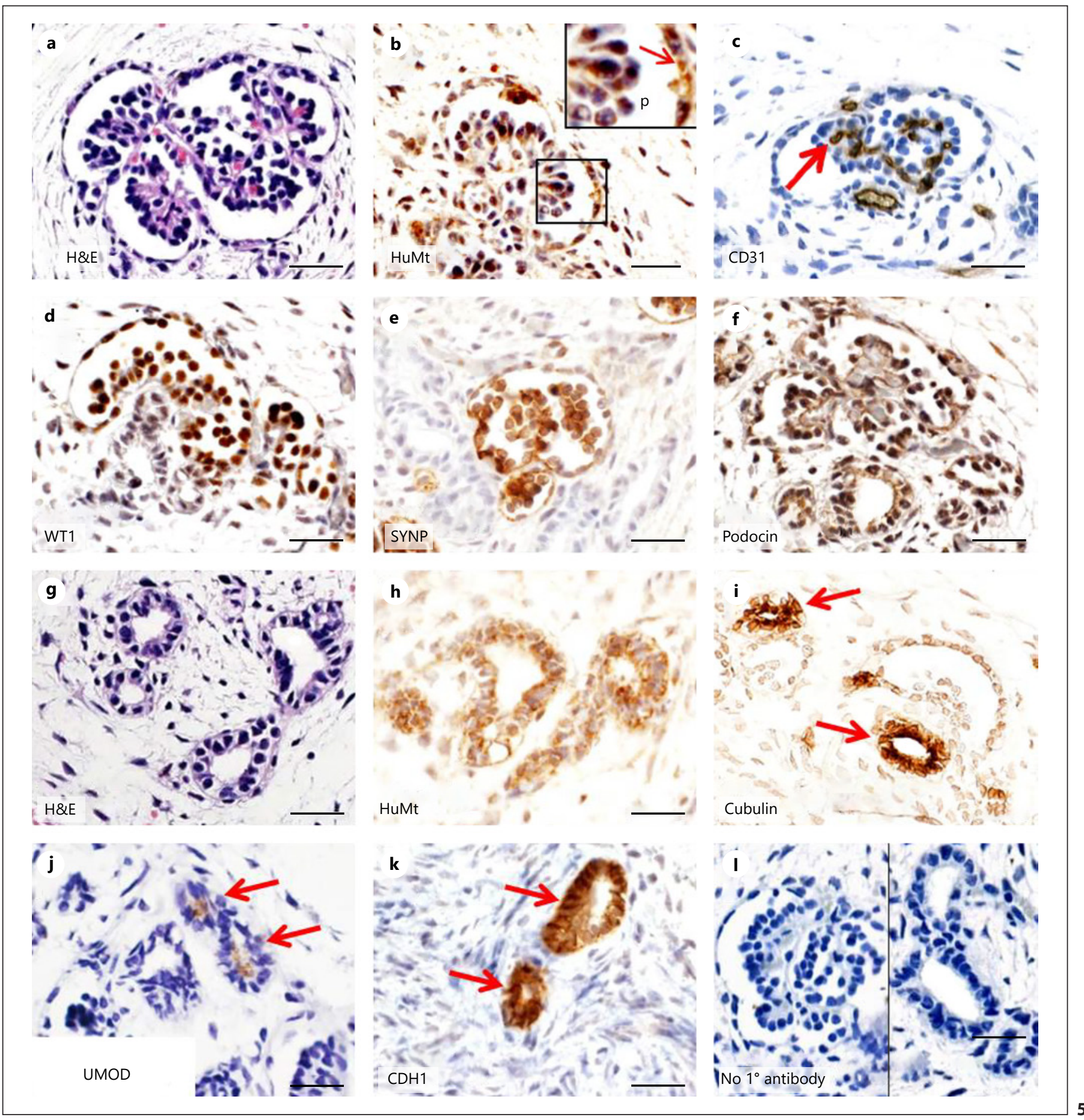


as were the kidney-like tissues. Quantification of histology (Fig. 6g-i) showed that chambers with AVFTs contained significantly ( $p=0.045)$ larger areas of human tissues (i.e., tubules plus glomeruli plus muscle-like cells) than chambers without AVFTs. There was, however, no significant ( $p=0.135)$ difference in amounts of "kidney tissues" (i.e., tubules plus glomeruli) between the 2 types of chamber.

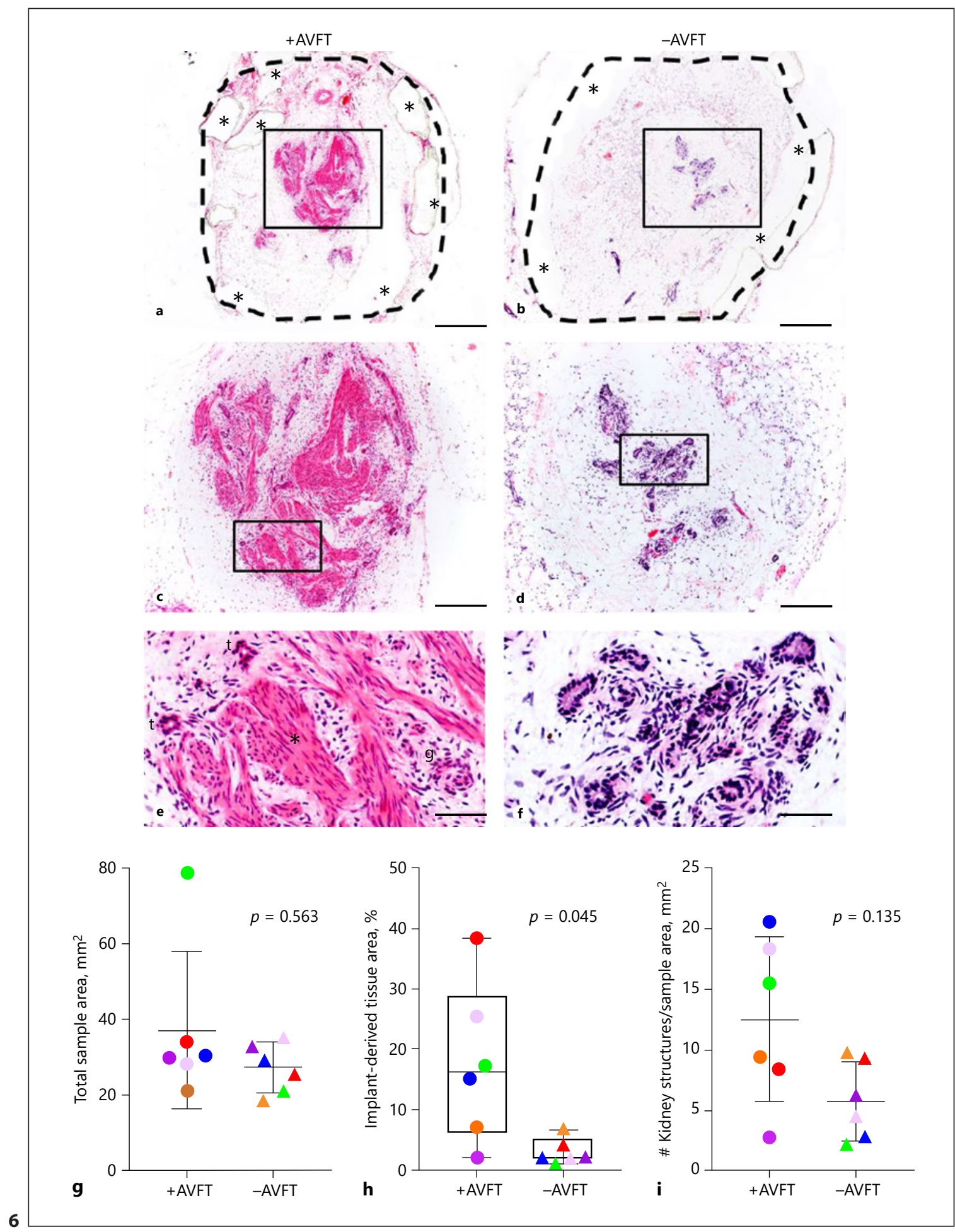

(For legend see next page.) 
We then probed sections with a panel of antibodies, comparing chambers containing AVFTs with those lacking AVFTs. Inside all chambers, tubules were detected, and they were positive for HuMt (Fig. 7a, b; see online suppl. Fig. 2 for higher resolution images). Muscle-like cells in chambers with AVFTs were also reactive to this antibody (Fig. 7a; online suppl. Fig. 2 for higher resolution image). Sparse small-calibre CD31-positive vessels of mouse but not human origin were noted in all chambers (Fig. 7c, d) but were not quantified. Tubules immunostained for CDH1 (Fig. 7e, f) and for hepatocyte nuclear factor 1B (Fig. 6g, h), a transcription factor expressed human fetal kidney tubules [19]. Some of these tubules appeared to be branched (Fig. 7e), a collecting duct characteristic. Tubules did not stain with cubilin or uromodulin antibodies, and only sparse rudimentary glomerular structures were seen (data not shown). In all chambers, loosely arranged stromal-like cells around tubules were positive for a-SMA (Fig. 7i, j), as were the prominent muscle-like cells that were located further away from the tubules in chambers containing AVFTs (Fig. 7i). These muscle-like cells immunostained prominently for transforming growth factor- $\beta 1$ (TGF- $\beta 1$ ) (Fig. 7k), while TGF- $\beta 1$ immunostaining appeared less prominent in chambers without AVFTs (Fig. 7l). No signal was obtained upon omission of primary antibodies (Fig. $7 \mathrm{~m}, \mathrm{n}$ ). Picrosirius red (PSR), a dye that binds to various matrix proteins including collagens [20], stained both the dense blocks of tissues around tubules in chambers with AVFTs as well as the loosely packed cells immediately surrounding tubules (Fig. 7o, p). Inspecting the same PSR-stained slides under polarized light, a technique more specific for collagens [20], revealed apparently more prominent signals around tubules in chambers with AVFTs versus those without AVFTs (online suppl. Fig. 3).

Fig. 6. Quantification of tissues within chambers implanted with human KPCs and harvested after 3 months. Histology sections stained with hematoxylin and eosin of chambers harvested 3 months after surgery. Bars are $500 \mu \mathrm{m}$ in $(\mathbf{a}, \mathbf{b}), 200 \mu \mathrm{m}$ in (c, d), and $50 \mu \mathrm{m}$ in $(\mathbf{e}, \mathbf{f})$. a Low power of cross section of chamber with an AVFT. The dotted line is drawn around the outside of the chamber and the asterisks indicate walls of the chamber. The inside of the chamber is dominated by pink tissues that on serial enlargements in (c, e) resolve into areas occupied by muscle-like cells. Tubule-like (t) and glomerular-like (g) structures are apparent in $\mathbf{e} . \mathbf{b}, \mathbf{d}, \mathbf{f}$ are a similar series of views of a chamber that had not received an AVFT. Note that some tubule-like structures are present but the zones of eosin staining muscle-like cells are not

\section{Discussion}

The results of the current study add a note of caution regarding the longer term in vivo fates of hPSC-derived KPCs and suggest that a period of increased vascularity may even enhance pathological differentiation. In the first set of experiments that we undertook, we proved that AVFTs could be reproducibly fashioned in the thighs of $S C I D /$ beige mice. When examined at 3 weeks after surgery, each such chamber contained an artery and vein with patent lumens. Having demonstrated the feasibility of this approach, we proceeded to undertake experiments in which hPSC-derived KPCs were placed inside AVFT chambers. When assessed at 3 weeks, KPCs implanted into chambers, either with or without AVFTs, formed glomeruli, containing capillary loops, and proximal-like tubules. Moreover, at this time point, AVFT chambers containing KPCs were more vascular than chambers in opposite thighs that contained KPCs but lacked AVFTs. This showed that the AVFT strategy had indeed created "vascularized bioreactors," at least at this time point. There was a non-significant tendency for more renal tissues in chambers with AVFTs. Nevertheless, when correlating the amount of renal tissue with the density of blood vessels, no statistically significant relationship was found. The latter observation indicates that this currently used AVFT strategy did not overtly enhance short-term differentiation of implanted cells into kidney tissues.

We considered the origin of the CD31 expressing vessels in the chambers implanted with human KPCs. In a previous study, we implanted hPSC (MAN13)-derived KPCs subcutaneously on the backs of SCID/beige mice [8]. In that report, we demonstrated that blood capillaries located inside the arising glomeruli were reactive with an antibody that detected human but not mouse CD31. In the current implant study, we were therefore surprised to

prominent. g Similar total areas used for quantification in chambers with AVFTs (left column) and those lacking AVFTs (right column); note that each mouse has a designated colour with the AVFT chamber shown as a circle and the opposite non-AVFT chamber shown as a triangle. $\mathbf{h}$ There was a significantly greater area of "implant derived tissue" (i.e., tubules plus glomeruli plus muscle-like cells) in chambers that had received AVFTs than in those that lacked AVFTs. $\mathbf{i}$ There was no significant difference between the 2 types of chamber in the percentage of areas occupied by "kidney structures" (i.e., tubules plus glomeruli but excluding muscle-like cells). KPC, kidney precursor cells; AVFT, arterial and venous flow-through. 

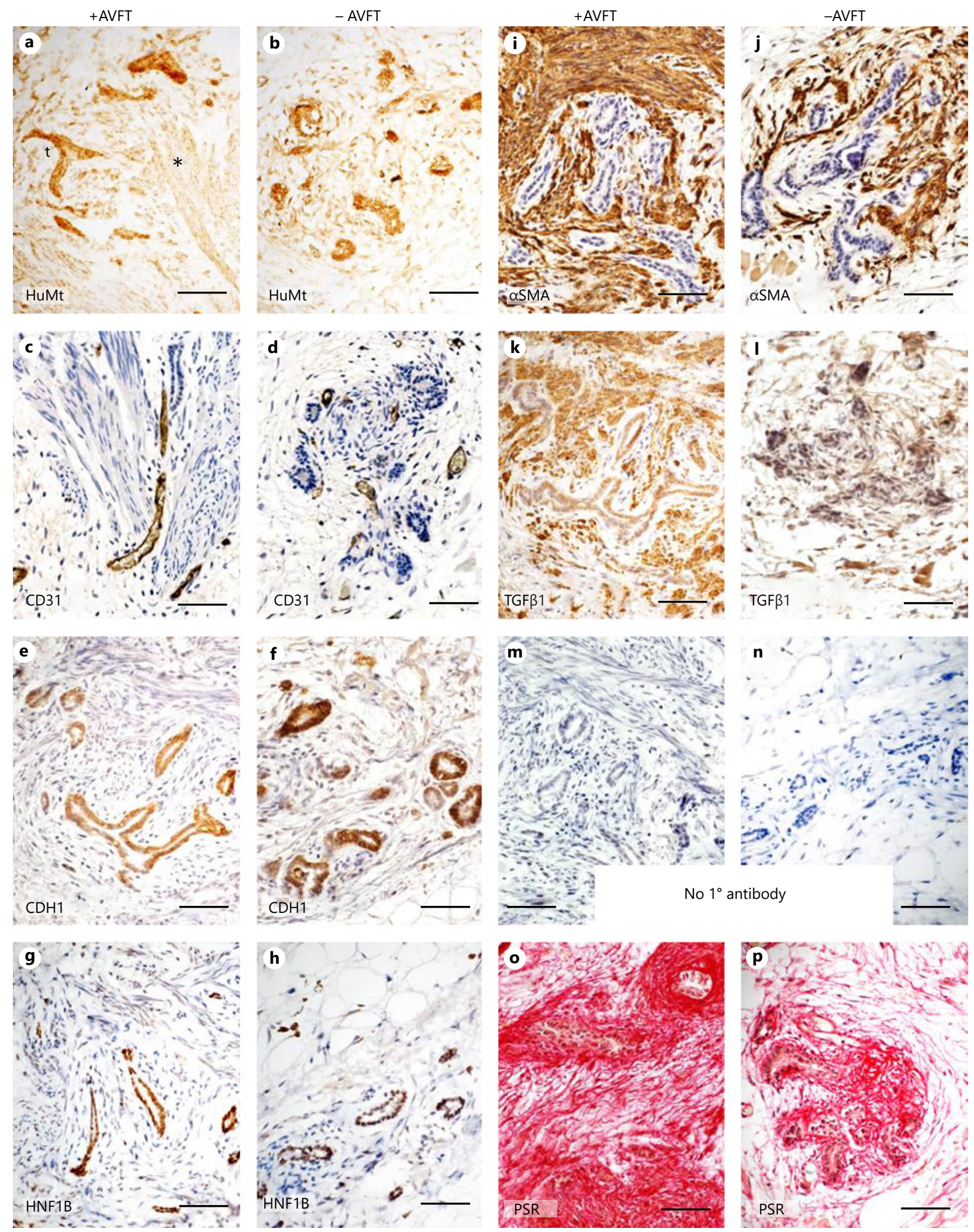

(For legend see next page.) 
reach a different conclusion, namely, that vessels in glomerular tufts appeared to be of mouse origin. The reasoning behind this conclusion was that vessels within implant chambers were detectable with an antibody reacting with mouse and human CD31 but not with an antibody specific for human CD31; the latter was shown to detect glomerular capillaries in the native human developing kidney. How can we reconcile the current results with those reported in subcutaneous implants [8]? One clue could be that there had been a change in composition of culture media. Indeed, STEMdiff ${ }^{\mathrm{TM}} \mathrm{APEL}^{\mathrm{TM}} 2$ medium was used instead of the discontinued STEMdiff ${ }^{\mathrm{TM}}$ APEL $^{\mathrm{TM}}$ medium, and the former lacks hybridoma extract. On the other hand, in the current study, we noted that CD31positive cells were detected around tubule-like structures that formed in tissue culture. This indicates that the human culture protocol we used can indeed generate cells with at least some endothelial characteristics. Therefore, we favour a different explanation that incorporates the idea that the origin of glomerular capillaries is highly context-dependent. A subset of cells located within native metanephric mesenchyme express endothelial molecular markers [21] and appear to have the potential to form glomerular capillaries, as occurs after the mouse metanephros is implanted into the cortex of neonatal mice kidneys [21]. On the other hand, nascent metanephric vessels are labile, and they tend to regress in normoxic organ culture [22]. Moreover, when mouse metanephric kidneys are explanted onto the chorioallantoic membrane, avian vessels invade the mouse kidney explant and colonize the differentiating murine glomerular tufts, seemingly to the exclusion of mouse endothelia $[21,23]$. In our current study, therefore, the "chamber implanted into thigh" scenario appears to favour mouse over hPSCderived vessels, analogous to the chorioallantoic membrane model described above.

Some of the implants were left for 3 months before they were harvested. Having seen a tendency for more

Fig. 7. Characterization of tissues within chambers implanted with human KPCs and harvested after 3 months. a, c, e, g, i, k, m, o are from chambers with AVFTs; the other frames are from chambers without AVFTs. All sections were counterstained with hematoxylin apart from $\mathbf{o}$ and $\mathbf{p}$. All bars are $50 \mu \mathrm{m}$. a, b HuMt immunostaining (brown); $t$ indicates a tubule and the asterisk is over the elongated muscle-like cells. See online suppl. Fig. 2 for higher power images. c, d Sparse CD31 immunostained (brown) vessels. e, f CDH1 immunostained (brown) tubules. g, h HNF1B immunostained (brown) tubules. i, j a-SMA immunostaining (brown) of both the stroma directly around tubules and, in the AVFT cham- renal tissues in implants with AVFTs at 3 weeks, we anticipated that the AVFT strategy might lead to an even more pronounced growth of differentiated kidney tissues by 3 months. At 3 months, however, chambers that had been implanted with KPCs contained tubules that, unlike the 3 week in vivo tubules, stained with neither cubilin nor uromodulin antibodies. Moreover, again in contrast to the 3-week implants, glomeruli appeared rudimentary and avascular. Therefore, we reason that the relatively normal kidney tissues seen at 3 weeks had de-differentiated after 3 months. In the subset of these chambers with AVFTs, prominent areas of muscle-like cells were also detected further away from tubules. The non-tubular cells were also reactive with the human mitochondrial antibody, so they must have arisen from implanted hPSCderived KPCs.

Congenital kidney malformations are the main cause of ESRD in children [24] and young adults [25]. A common phenotype is the dysplastic kidney, and its histology features primitive ducts surrounded by metaplastic smooth muscle-like cells, together with a scarcity of glomeruli [26-28]. It has been postulated that renal dysplasia arises from impaired induction between the ureteric bud and the metanephric mesenchyme [26], precursors that will normally form collecting ducts and nephrons, respectively. An upregulation of the TGF- $\beta$ axis has been described in human renal dysplastic tissues, and application of TGF- $\beta 1$ induces trans-differentiation of human kidney dysplastic epithelia to elongated cells expressing a-SMA [29]. Moreover, exposure of explanted murine metanephric kidneys [30] and embryonic ureters [31] to TGF- $\beta 1$ elicits dysgenesis of each tissue. Thus, the phenotypes of abnormal tissues generated from human KPCs in the current study have some similarities to the histological signature of human renal dysplasia. TGF $\beta 1$, however, is just one secreted protein implicated in normal and abnormal kidney development, as well as in renal trans-differentiation and scarring. In future, it would be useful to 
seek changes in a variety of other such proteins such as bone morphogenetic protein 7 and CD44/hyaluran that impact on similar processes $[32,33]$ in this regard, proteomic analyses of matrix-associated proteins [34] from tissues harvested from the chambers could be used.

In the current set of implants, we did not observe metaplastic cartilage, another feature of human renal dysplasia [26]. In contrast, we reported that areas of cartilage were detected in subcutaneous implants of this same hPSC on the backs of SCID mice [8], suggesting that the specific type of non-renal mesodermal differentiation may be influenced by different implant settings.

Our current study is also consistent with the conclusion that pathological differentiation may follow a period of increased vascularity. At first glance, this sounds counterintuitive because, for example, kidney fibrosis in postnatal organs is associated with a progressive depletion of vessels $[35,36]$. On the other hand, some of the same molecular effectors of angiogenesis may also enhance scarring [37]. It is also possible that over-abundant externally derived vessels plus a lack of endogenous kidney vessels, as observed in the current study, may be detrimental. Another explanation for the generation of aberrant tissue could be considered apart from altered vascularity per se. Perhaps, the differentiation potential of the implanted KPCs was, in some yet-to-be-determined manner, also modified by local cellular niches conferred by multiple factors, for example, the AVFT, nearby host tissues such as thigh muscles, or the plastic $3 \mathrm{D}$ printed chamber. These explanations may at least in part explain why we did not detect aberrant differentiation after hPSC-derived KPCs were implanted subcutaneously into SCID/beige mice $[8,11]$. In those experiments, although cells were also embedded in Matrigel ${ }^{\mathrm{TM}}$, neither chambers nor AVFTs were employed.

\section{Materials and Methods}

\section{Chamber Production}

Chambers were designed using 3D CAD software (Autodesk Inventor 2015, student edition) as hemispheres with 0.5 -mm-thick walls, and 1-mm-diameter pores distributed uniformly on the surface of the chamber. The 2 parts of the chamber were joined by a central pillar. The intact chamber was kidney-shaped and was 5.0 $\mathrm{mm}$ long with a maximum diameter of $3.5 \mathrm{~mm}$. Chambers were printed via stereolithography in a Form $23 \mathrm{D}$ printer (Formlabs, Somerville, MA, USA) with a biocompatible photopolymer resin material (Dental SG resin material; Formlabs, UK), a liquid photopolymer resin. Following print completion and curing and sterilising in UV light for $30 \mathrm{~min}$, all support material was removed, and the chambers were washed in distilled sterile water and $0.1 \%$ betadine before implantation.

\section{Generation of Human KPCs in Culture}

MAN13 hPSCs [38] were grown on 24-well plates coated with $5 \mu \mathrm{g} / \mathrm{mL}$ recombinant human vitronectin (rhVTN-N, Life Technologies, \#A14700) in mTeSR1 (StemCell Technologies, \#5850) with the medium changed every 2 days. The cells were passaged by treatment of the cultures with $0.5 \mathrm{~mm}$ EDTA solution, $\mathrm{pH} 8$ (Invitrogen, \#15575-038; diluted in PBS) and replating the cells in mTeSR1 medium, containing $5 \mathrm{nM}$ ROCK inhibitor, Y-27632 (Tocris, \#1254) for $24 \mathrm{~h}$. For differentiation, hPSCs were plated on vitronectin-coated plates, at a density of 18,000 cells $/ \mathrm{cm}^{2}$ in mTeSR1 medium containing $10 \mu \mathrm{m}$ Y-27632. The following day, the medium was replaced with STEMdiff ${ }^{\mathrm{TM}} \mathrm{APEL}^{\mathrm{TM}} 2$ Medium (STEMCELL Technologies) containing 8 MM CHIR-99021 (Tocris, \#4423) for 3 days, followed by STEMdiff ${ }^{\mathrm{TM}}$ APEL $^{\mathrm{TM}} 2$ Medium supplemented with $200 \mathrm{ng} / \mathrm{mL}$ FGF9 (Peprotech, \#100-23) and $1 \mu \mathrm{g}$ / $\mathrm{mL}$ heparin (Sigma, \#3149) for a further 10 days. Subsequently, the cells were cultured in basal STEMdiff ${ }^{\mathrm{TM}}$ APEL $^{\mathrm{TM}} 2$ Medium, which was changed daily. Immunocytochemistry is detailed in the online suppl. Materials and Methods.

\section{Animal Surgery}

Procedures were approved by the University of Manchester Ethical Committee and the UK Home Office project licence 70/8686. Animal work was undertaken under Animal Research: Reporting of In Vivo Experiments guidelines (https://www.nc3rs. org.uk/arrive-guidelines). Female SCID/beige mice (Harlan Laboratories) aged 10-12 weeks were anaesthetized by isoflurane (Abbot Laboratories Ltd., UK) as follows: induction at $4 \mathrm{~L} / \mathrm{min}$ oxygen with $4 \%$ isoflurane and then maintained at $2 \mathrm{~L} / \mathrm{min}$ oxygen with $2 \%$ isoflurane during the rest of the procedure. Mice were immobilized on their posterior, and the hind limb was shaved up to the arcuate line. Under an operating microscope (Leica MZ7.5; Leica Microsystems, Switzerland), the femoral artery and vein between the junction of the tibial veins to the inguinal ligament were carefully exposed from surrounding tissues with curved microscissors (SDC-15; Mercian Surgical, UK) and forceps (Dumont \#5/45; Fine Science Tools, Germany). Vessels feeding into the vein were cauterized (Bovie, Clearwater, FL, USA) or ligated with 11-0 sutures (Ethilon, Ethicon, UK). The femoral artery and vein were carefully separated from adventitia to allow mobilisation to allow for placement of the 3D printed plastic chamber around the vessels. These vessels, which comprised the AVFT, passed through the chamber around the central pillar, which connected the 2 halves. A control chamber without an AVFT was secured into space created in the inguinal region of the opposite thigh. In some experiments, before they were closed and implanted, chambers were populated with 2 million human KPCs placed as a pellet in $30 \mu \mathrm{L}$ of Matrigel ${ }^{\mathrm{TM}}$. The chamber was secured into space created in the inguinal region with 8-0 sutures (Ethilon, B.Braun, Germany), and the skin was closed with 2 layers of 8-0 sutures. Further controls contained Matrigel and no cells were also performed. Analgesia $(0.1 \mathrm{mg} / \mathrm{kg}$, Vetergesic; Alstoe Animal Health) was administered, and the animals were allowed to recover with $2 \mathrm{~L} / \mathrm{min}$ oxygen. All animals underwent the procedure without complications such as chamber extrusion, infection, or haemorrhage.

\section{Histology of Implanted Tissues}

Each harvested chamber was fixed in $4 \%$ paraformaldehyde, embedded in paraffin, and sectioned throughout its length at $5 \mu \mathrm{m}$. Sections were dewaxed and rehydrated and used for staining and 
the quantification of areas occupied by particular cell types. For the latter analyses, every 15th slide was used and the total quantified area comprised the inside of the chamber plus the small areas in the perforations of each chamber. Tissues external to the chamber wall were excluded from these analyses. For specific stains, see online suppl. Materials and Methods.

\section{Human Embryonic Kidneys}

Human tissues, collected after consent and ethical approval (REC 08/H0906/21 + 5), were provided by the MRC and Wellcome Trust Human Developmental Biology Resource (http://www. hdbr.org/), as described previously [31].

\section{Statistical Methods}

Analyses were performed using GraphPad Prism software (GraphPad Prism v.7) (https://www.graphpad.com/scientificsoftware/prism/). Data sets in each experimental group were subjected to the Shapiro-Wilk test to determine whether or not they were compatible with a normal distribution. In the former cases, data were shown as mean $\pm \mathrm{SD}$, whereas non-parametric sets were shown as the median with the interquartile and absolute ranges. We compared results from each implant with an AVFT with its counterpart lacking an AVFT in the opposite thigh of the same mouse. Thus, parametric (2-tailed $t$ test) or non-parametric (2-tailed Wilcoxon) paired analyses were applied, as appropriate. Differences were considered statistically significant when $p<0.05$ but, for clarity, exact $p$ values are given in graphs of the data in the Results section.

\section{Acknowledgements}

We acknowledge grant support as follows: the Medical Research Council (MR/K026739/1 and MR/T016809/1), Kidney Research UK (RP_021_20170302 and JFS/RP/008/20160916), the Engineering and Physical Sciences Research Council (EP/ L012022/1), Kidneys for Life (2018 pump priming grants), the Royal College of Surgeons of Edinburgh Support Grant, and the University Hospital of South Manchester Burns and Plastics Endowment Fund. The University of Manchester Bioimaging Facility microscopes used in this study were purchased with support from the Biotechnology and Biological Sciences Research Council, the Wellcome Trust, and the University of Manchester Strategic Fund. We thank Bioimaging Facility staff for their help with the microscopy. We thank Andrew Weightman and Edward Whitehouse (Department of Mechanical, Aerospace and Civil Engineering, University of Manchester) for technical input.

\section{Statement of Ethics}

Procedures were approved by the University of Manchester Ethical Committee and the UK Home Office project licence 70/8686. Animal work was undertaken under Animal Research: Reporting of In Vivo Experiments guidelines (https://www.nc3rs. org.uk/arrive-guidelines).

\section{Conflict of Interest Statement}

The authors have no conflict of interests to declare.

\section{References}

1 Kimber SJ, Woolf AS. From human pluripotent stem cells to functional kidney organoids and models of renal disease. Stem Cell Investig. 2018;5:20.

2 Little MH, Combes AN. Kidney organoids: accurate models or fortunate accidents. Genes Dev. 2019;33(19-20):1319-45.

3 Woolf AS. Growing a new human kidney. Kidney Int. 2019;96(4):871-82.

4 Bonventre JV. Kidney organoids-a new tool for kidney therapeutic development. Kidney Int. 2018;94(6):1040-2.

5 Takasato M, Er PX, Becroft M, Vanslambrouck JM, Stanley EG, Elefanty AG, et al. Directing human embryonic stem cell differentiation towards a renal lineage generates a self-organizing kidney. Nat Cell Biol. 2014; 16(1):118-26.

6 Takasato M, Er PX, Chiu HS, Maier B, Baillie GJ, Ferguson C, et al. Kidney organoids from human iPS cells contain multiple lineages and model human nephrogenesis. Nature. 2015; 536(7615):238-8.

7 Taguchi A, Nishinakamura R. Higher-order kidney organogenesis from pluripotent stem cells. Cell Stem Cell. 2017;21(6):730-46.e6.
8 Bantounas I, Ranjzad P, Tengku F, Silajdžić E, Forster D, Asselin MC, et al. Generation of functioning nephrons by implanting human pluripotent stem cell-derived kidney progenitors. Stem Cell Reports. 2018;10(3): 766-79.

$9 \mathrm{Wu} \mathrm{H}$, Uchimura K, Donnelly EL, Kirita Y, Morris SA, Humphreys BD. Comparative analysis and refinement of human PSC-derived kidney organoid differentiation with single-cell transcriptomics. Cell Stem Cell. 2018;23(6):869-81.e8.

10 Przepiorski A, Sander V, Tran T, Hollywood JA, Sorrenson B, Shih JH, et al. A simple bioreactor-based method to generate kidney organoids from pluripotent stem cells. Stem Cell Reports. 2018;11(2):470-84.

11 Bantounas I, Silajdžić E, Woolf AS, Kimber SJ. Formation of mature nephrons by implantation of human pluripotent stem cell-derived progenitors into mice. Methods Mol Biol. 2020;2067:309-22.

12 Sharmin S, Taguchi A, Kaku Y, Yoshimura Y, Ohmori T, Sakuma T, et al. Human induced pluripotent stem cell-derived podocytes mature into vascularized glomeruli upon experi- mental transplantation. J Am Soc Nephrol. 2016;27(6):1778-91.

13 van den Berg CW, Ritsma L, Avramut MC, Wiersma LE, van den Berg BM, Leuning DG, et al. Renal subcapsular transplantation of PSC-derived kidney organoids induces neovasculogenesis and significant glomerular and tubular maturation in vivo. Stem Cell Reports. 2018;10(3):751-65.

14 Fine LG. Are current strategies for building a human kidney misguided? Speculative alternatives. J Am Soc Nephrol. 2018;29(12):2780-2.

15 Fiegel HC, Pryymachuk G, Rath S, Bleiziffer $\mathrm{O}$, Beier JP, Bruns H, et al. Foetal hepatocyte transplantation in a vascularized AV-loop transplantation model in the rat. J Cell Mol Med. 2010;14(1-2):267-74.

16 Yap KK, Yeoh GC, Morrison WA, Mitchell GM. The vascularised chamber as an in vivo bioreactor. Trends Biotechnol. 2018;36(10): 1011-24.

17 Wong R, Donno R, Leon-Valdivieso CY, Roostalu U, Derby B, Tirelli N, et al. Angiogenesis and tissue formation driven by an arteriovenous loop in the mouse. Sci Rep. 2019; 9(1):10478. 
18 Cronin KJ, Messina A, Knight KR, CooperWhite JJ, Stevens GW, Penington AJ, et al. New murine model of spontaneous autologous tissue engineering, combining an arteriovenous pedicle with matrix materials. Plast Reconstr Surg. 2004;113(1):260-9.

19 Kolatsi-Joannou M, Bingham C, Ellard S, Bulman MP, Allen LI, Hattersley AT, et al. Hepatocyte nuclear factor-1beta: a new kindred with renal cysts and diabetes and gene expression in normal human development. J Am Soc Nephrol. 2001;12(10):2175-80.

20 Rittié L. Method for picrosirius red-polarization detection of collagen fibers in tissue sections. Methods Mol Biol. 2017;1627:395-407.

21 Loughna S, Hardman P, Landels E, Jussila L, Alitalo K, Woolf AS. A molecular and genetic analysis of renal glomerular capillary development. Angiogenesis. 1997;1(1):84-101.

22 Loughna S, Yuan HT, Woolf AS. Effects of oxygen on vascular patterning in Tie1/LacZ metanephric kidneys in vitro. Biochem Biophys Res Commun. 1998;247(2):361-6.

23 Sariola H, Timpl R, von der Mark K, Mayne R, Fitch JM, Linsenmayer TF, et al. Dual origin of glomerular basement membrane. Dev Biol. 1984;101(1):86-96.

24 Hamilton AJ, Braddon F, Casula A, Lewis M, Mallett T, Marks SD, et al. UK renal registry 19th annual report: chapter 4 demography of the UK paediatric renal replacement therapy population in 2015. Nephron. 2017;137(Suppl 1):103-16.
25 Neild GH. What do we know about chronic renal failure in young adults? II. Adult outcome of pediatric renal disease. Pediatr Nephrol. 2009;24(10):1921-8.

26 Potter EL. Normal and abnormal development of the kidney. Year Book Medical Publishers; 1972.p. 1-305. ISBN-10: 0815167636.

27 Woolf AS, Price KL, Scambler PJ, Winyard PJ. Evolving concepts in human renal dysplasia. J Am Soc Nephrol. 2004;15(4):998-1007.

28 Liapis H, Yu H, Flath A, Steinhardt GF. Increased expression of platelet-derived growth factor A and collagenous matrix proteins in congenital multicystic renal dysplasia. Nephron. 1997;76(2):161-70.

29 Yang SP, Woolf AS, Yuan HT, Scott RJ, Risdon RA, O'Hare MJ, et al. Potential biological role of transforming growth factor-betal in human congenital kidney malformations. Am J Pathol. 2000;157(5):1633-47.

30 Rogers SA, Ryan G, Purchio AF, Hammerman MR. Metanephric transforming growth factor-beta 1 regulates nephrogenesis in vitro. Am J Physiol. 1993;264(6 Pt 2):F996-1002.

31 Lopes FM, Roberts NA, Zeef LA, Gardiner NJ Woolf AS. Overactivity or blockade of transforming growth factor- $\beta$ each generate a specific ureter malformation. J Pathol. 2019; 249(4):472-84.
32 Pohl M, Sakurai H, Stuart RO, Nigam SK. Role of hyaluronan and CD44 in in vitro branching morphogenesis of ureteric bud cells. Dev Biol. 2000;224:312-325.

33 Rampanelli E, Rouschop K, Teske GJD, et al. CD44v3-v10 reduces the profibrotic effects of TGF- $\beta 1$ and attenuates tubular injury in the early stage of chronic obstructive nephropathy. Am J Physiol Renal Physiol. 2013;305: F1445-F1454.

34 Randles MJ, Woolf AS, Huang JL, et al. Genetic background is a key determinant of glomerular extracellular matrix composition and organization. J Am Soc Nephrol. 2015;26: 3021-3034

35 Kang DH, Kanellis J, Hugo C, Truong L, Anderson S, Kerjaschki D, et al. Role of the microvascular endothelium in progressive renal disease. J Am Soc Nephrol. 2002;13(3):80616.

36 Kang DH, Johnson RJ. Vascular endothelial growth factor: a new player in the pathogenesis of renal fibrosis. Curr Opin Nephrol Hypertens. 2003;12(1):43-9.

37 Long DA, Price KL, Ioffe E, Gannon CM, Gnudi L, White KE, et al. Angiopoietin-1 therapy enhances fibrosis and inflammation following folic acid-induced acute renal injury. Kidney Int. 2008;74(3):300-9.

38 Ye J, Bates N, Soteriou D, Grady L, Edmond C, Ross A, et al. High quality clinical grade human embryonic stem cell lines derived from fresh discarded embryos. Stem Cell Res Ther. 2017;8(1):128. 